\title{
Effects of patient factors on noninvasive liver stiffness measurement using acoustic radiation force impulse elastography in patients with chronic hepatitis $C$
}

Sheng-Hung Chen ${ }^{1,2}$, Yu-Fen Li ${ }^{3}$, Hsueh-Chou Lai ${ }^{1,4}$, Jung-Ta Kao ${ }^{1,2}$, Cheng-Yuan Peng ${ }^{1,5^{*}}$, Po-Heng Chuang ${ }^{1,2}$, Wen-Pang Su ${ }^{1,2}$ and I-Ping Chiang ${ }^{5,6}$

\begin{abstract}
Background: Previous research has shown variation in the effects of patient factors, including hepatic necroinflammatory activity, on liver stiffness measurement (LSM). This prospective study attempts to identify explanatory factors for LSM in patients with chronic hepatitis $C(C H C)$ using acoustic radiation force impulse (ARFI) technology.

Methods: A cohort of 127 Taiwanese patients with CHC underwent ARFI LSM and immediate percutaneous liver biopsy. This study compares the concurrent diagnostic performances of LSM and FibroTest using receiver operating characteristic (ROC) curves. Three multiple linear regression models were used to evaluate the significance of concurrent patient factors in explaining LSM.

Results: To classify METAVIR fibrosis (F) stages, the areas under ROC curves (AUCs) were ARFI LSM, 0.847 (95\% confidence interval (CI), 0.779-0.914) and FibroTest, 0.823 (95\% Cl, 0.748-0.898), for F1 versus F2-4; ARFI LSM, 0.902 (95\% Cl, 0.835-0.970) and FibroTest, 0.812 (95\% Cl, 0.735-0.888), for F1-2 versus F3-4; ARFI LSM, 0.831 (95\% Cl, $0.723-0.939)$ and FibroTest, 0.757 ( $95 \% \mathrm{Cl}, 0.648-0.865)$, for F1-3 versus F4. After adjusting for other demographic and biological covariates, biochemical and histological necroinflammatory factors consistently explained LSM. Factors included serum alanine aminotransferase (ALT)/upper limit of normal (ULN) categories (model $R^{2}=0.661$, adjusted $\left.R^{2}=0.629\right)$, ActiTest A scores $\left(R^{2}=0.662\right.$, adjusted $\left.R^{2}=0.636\right)$, and METAVIR activity (A) grades $\left(R^{2}=0.651\right.$, adjusted $\left.R^{2}=0.620\right)$. METAVIR F stages, body mass index, and platelet count were also independently associated with LSM. Necroinflammatory degrees, including ALT/ULN, ActiTest A scores, and METAVIR A grades, explained the false positivity of liver fibrosis staging using ARFI LSM.

Conclusions: The degree of hepatic necroinflammatory activity independently and significantly exaggerated liver fibrosis evaluation using ARFI LSM. However, comparisons with concurrent FibroTest indicate that ARFI LSM may be a promising alternative, or adjunctive single indicator, for liver fibrosis evaluation in patients with CHC.
\end{abstract}

Keywords: Liver fibrosis, Cirrhosis, Acoustic radiation force impulse, FibroTest, ActiTest, Chronic hepatitis C

\footnotetext{
* Correspondence: cypeng@mail.cmuh.org.tw

'Division of Hepatogastroenterology, Department of Internal Medicine, China Medical University Hospital, No 2 Yuh-Der Road, Taichung 40402, Taiwan

${ }^{5}$ School of Medicine, China Medical University, Taichung 40402, Taiwan

Full list of author information is available at the end of the article
} 


\section{Background}

Hepatitis $\mathrm{C}$ virus (HCV) infection is a leading cause of cirrhosis and hepatocellular carcinoma (HCC) worldwide [1]. In the current era of antiviral and antifibrotic treatments, clinical and research demands are increasing significantly worldwide for noninvasive surveillance methods for liver fibrosis. These methods are necessary to evaluate the progression and regression of liver fibrosis [2-6].

Recent investigations have proposed liver stiffness measurement (LSM) using acoustic radiation force impulse (ARFI) elastography as a novel, reliable, and accurate noninvasive solution for evaluating liver fibrosis [7-15]. However, the cutoff variability in previous studies may be the result of a limited number of samples with diverse hepatitis etiologies and variable sample characteristics, including demographic and biological covariates, ethnicity, and prolonged intervals between liver biopsy and LSM. In several of these studies, the time intervals between biopsy and LSM were as long as 6 to 15 months $[8,12,13]$. These may have affected the LSM results because of potential histological progression and the dynamic fibrogenic process.

Although previous studies [7,8,10,12-15] have implemented concurrent FibroScan (Echosens, Paris, France) and ARFI, few studies [8,9] have compared [16] the diagnostic performances or discrimination capabilities of ARFI and FibroTest (BioPredictive, Paris, France) in evaluating liver fibrosis. Local LSM spectra using ARFI technology are still lacking for Taiwanese patients with chronic hepatitis $\mathrm{C}(\mathrm{CHC})$.

In addition to several factors related to the stiffness measurement itself [14,17-19], several patient factors, including hepatic necroinflammatory activity $[12,13,15$, 20-30], cholestasis [31,32], and cardiac congestion [33], affect the LSM values in liver fibrosis evaluation. Therefore, independent significant explanatory patient factors should be evaluated comprehensively with caution when interpreting or fine-tuning the results of ARFI LSM. However, previous studies have shown variation in the effects of patient factors, including hepatic necroinflammatory activities, on LSM using FibroScan or ARFI [12,13,28,32]. Despite the wide use of hepatic elastography, few studies [12,29,30] have correlated ARFI LSM with concurrent histological steatosis and necroinflammatory categories, including the METAVIR activity (METAVIR A) grades, and not merely blood test results. Few studies have measured the necroinflammatory degrees using ActiTest (BioPredictive, Paris, France) [34].

To date, clinically applicable noninvasive tests do not focus on the exact staging of disease, but rather on dividing patients into categories of milder versus more significant fibrosis and cirrhosis [5]. The adjusted effects of METAVIR fibrosis (METAVIR F) stages on ARFI LSM, and the potential limitations of ARFI LSM (especially the false positivity in liver fibrosis staging) also have yet to be fully elucidated.

This prospective study examines the contributions of concurrent patient factors, and especially the histological covariates, to ARFI LSM. This study also examines the adjusted effects of METAVIR $F$ stages on ARFI LSM, with the purpose of evaluating diagnostic performance using the simple and noninvasive single indicator ARFI LSM as compared with concurrent FibroTest. Finally, this study identifies the optimal real time ARFI LSM cutoff values for liver fibrosis staging in Taiwanese patients with $\mathrm{CHC}$.

\section{Methods}

\section{Patients}

From November 2010 to October 2011, a cohort of 142 consecutive Taiwanese patients participated in this prospective, operator-blind study. The participants were referred to the liver center for percutaneous liver biopsy prior to the initiation of standard of care (SOC) for $\mathrm{CHC}$. This study defines $\mathrm{CHC}$ as positive serum antiHCV antibody (Abbott Laboratories, Abbott Park, Illinois, USA) for more than six months with the presence of serum HCV RNA (Cobas Amplicor HCV Monitor 2.0; Roche Diagnostics, Branchburg, New Jersey, USA).

Five patients refused, or were contraindicated for, percutaneous liver biopsy. Thus, a total of 137 patients underwent percutaneous liver biopsy within one hour of receiving blood tests (including those for FibroTest) and stiffness measurements, preceded by three hours of fasting [35].

Patients with any of the following conditions or history were excluded from the sample: interferon or nucleos $(\mathrm{t})$ ide analogue treatment, exposure to hepatotoxic drugs or chemicals, primary biliary cirrhosis, primary sclerosing cholangitis, Wilson's disease, autoimmune hepatitis, alcoholic liver disease (ALD), hepatitis B virus (HBV) coinfection, human immunodeficiency virus (HIV) coinfection, liver abscess, acute hepatitis, extrahepatic cholestasis $[31,32]$, severe hemolysis, Gilbert's syndrome with high unconjugated hyperbilirubinemia, autoimmune disorders, myeloproliferative disorders, thalassemias, schistosomiasis, major abdominal surgery, cardiac congestion [33], blood product transfusion within the previous 30 days, pregnancy, liver cancer, serum creatinine higher than 221 umol/L (2.5 mg/dL), hepatic encephalopathy, refractory ascites [36], and variceal bleeding.

This study conforms to the Helsinki Declaration of 1975 and was approved by the Institutional Review Board of China Medical University Hospital (CMUH IRB No. DMR99-IRB-240). All individuals provided written informed consent prior to study enrollment. 


\section{FibroTest and ActiTest}

Serum markers including $\alpha 2$-macroglobulin, alanine aminotransferase (ALT), apolipoprotein A1, total bilirubin, $\gamma$-glutamyl transpeptidase (GGT) and haptoglobin were tested in the same laboratory, and results were then sent to www.biopredictive.com to determine a measure of liver fibrosis (FibroTest $\mathrm{F}$ score) and necroinflammatory activity (ActiTest A score) using patented artificial intelligence algorithms $[37,38]$.

\section{ARFI LSM}

ARFI technology [39] was integrated into a conventional ultrasound system (Acuson S2000 with a Siemens 4C1 curved array, $4.00 \mathrm{MHz}$ for B-mode, $2.67 \mathrm{MHz}$ for push pulses and 3.08 MHz for detection pulses; Siemens Medical Solutions, Mountain View, California, USA). The detection pulses measured the shear wave velocity (SWV), which was considered to directly relate to tissue stiffness.

All ARFI stiffness measurements were performed by the same hepatologist, who was experienced in digestive system ultrasonography and blinded to the patient data. The right lobe [17] of the liver (Couinaud segments VIII, V, VII, VI) was approached intercostally [18], with the patient lying in a dorsal decubitus position [12] with both arms above the head and holding their breath during virtual touch quantification (VTQ) measurements. During each measurement, the region of interest (ROI) $(10 \times 5 \mathrm{~mm})$ was placed at the middle of the acoustic field, avoiding large blood vessels and biliary tracts.

SWV results were recorded in meters per second $(\mathrm{m} / \mathrm{s})$ and stored as jpg image files. Each patient received 10 successful LSMs (failed measurements were defined as SWV = "x.xx m/s") [40]. Reliable cases were defined as those with an interquartile range (IQR) of less than $30 \%$ of the median of 10 successful LSMs, and a successful rate of LSMs greater than $60 \%$. Other cases were deemed unreliable and excluded $[9,10,19]$.

\section{Histology}

Senior hepatologists performed the percutaneous right lobe liver biopsy. All biopsy specimens were interpreted by an expert pathologist blinded to the results of LSMs and patient data. Biopsy specimens at least $15 \mathrm{~mm}$ in length containing at least five portal tracts were defined adequate [6]. Liver fibrosis and necroinflammatory activity were staged and graded using the METAVIR scoring system: F0, no fibrosis; F1, portal fibrosis without septa; F2, portal fibrosis with a few septa; F3, numerous septa without cirrhosis; and F4, cirrhosis; A0, none; A1, mild; A2, moderate; and A3, severe necroinflammatory activity [41]. Steatosis categories were S0, no steatosis; S1, 1 to $5 \%$ (percentage of hepatocytes containing visible macrovesicular steatosis); S2, 6 to $32 \%$; S3, 33 to $66 \%$; and S4, 67 to $100 \%$ [42].

\section{Statistical analysis}

The intraobserver correlations of ARFI LSM were evaluated using an intraclass correlation coefficient (ICC) for independent LSMs performed on two separate occasions in 20 patients by the same hepatologist with experience in 50 training cases.

Spearman's rank correlation was used to evaluate the significance of correlations between continuous variables and liver fibrosis stages. Intergroup differences were analyzed using Fisher's exact test for proportions, and the Student's $t$-test or one-way ANOVA for continuous variables, when appropriate.

Receiver operating characteristic (ROC) curves were used to optimize the cutoff values [43] and evaluate diagnostic performance.

After univariate linear regression, variables with a $P$ value of less than .25 were included in the subsequent stepwise multiple linear regression modelling. In addition to the two potential confounders of age and sex, the three final multiple linear regressions included variables maximizing the adjusted $R^{2}$ in each stepwise regression to identify significant independent explanatory factors for LSM. Four final multiple linear regression models were used to delineate the necroinflammatory effects on LSM after adjusting for serum ALT levels (model estimates are reported in the discussion section), ALT/upper limit of normal (ULN, $40 \mathrm{IU} / \mathrm{L}$ ) categories (model 1), ActiTest A scores (model 2) and METAVIR A grades (model 3). Binary logistic regression was also used to examine the necroinflammatory effects on false positivity in liver fibrosis evaluation using ARFI LSM in patients with METAVIR F1, F2 and F3.

Data were analyzed using SPSS version 17.0 for Microsoft Windows (SPSS, Chicago, Illinois, USA). A twosided $P$ value of $<.05$ indicated statistical significance.

\section{Results}

\section{Patients}

Among the 137 patients, this study excluded 2 patients with liver cancer (both HCC), 2 with ALD, 1 with end stage renal disease, 2 with unreliable LSM results, and 3 with inadequate specimen quality. Thus, 127 patients entered the complete analysis.

Age, serum ALT, total bilirubin, HCV RNA, international normalized ratio (INR) of prothrombin time, platelet count, liver SWV, and F score of FibroTest differed significantly between the non-cirrhotic and cirrhotic groups (Table 1). The serum ALT levels ranged from 14 to $488 \mathrm{IU} / \mathrm{L}$, and Actitest A scores ranged from 0.04 to 0.97 . Spearman's rank correlation coefficients 


\section{Table 1 Patient characteristics}

\begin{tabular}{|c|c|c|c|}
\hline \multirow[t]{2}{*}{ Variable } & \multirow{2}{*}{$\frac{\text { METAVIR F0-3 }}{n=109}$} & \multirow{2}{*}{$\frac{\text { METAVIR F4 }}{n=18}$} & \multirow[t]{2}{*}{$P$ value } \\
\hline & & & \\
\hline Age, year & $51.6(1.2)$ & $62.7(1.5)$ & $<.001$ \\
\hline Gender (n) & & & 1.000 \\
\hline Male & 51 & 8 & \\
\hline Female & 58 & 10 & \\
\hline $\mathrm{BMI}, \mathrm{kg} / \mathrm{m}^{2}$ & $24.64(0.33)$ & $24.02(0.79)$ & .477 \\
\hline HCV genotype (n) & & & 1.000 \\
\hline 1 & 53 & 9 & \\
\hline Non-1 & 56 & 9 & \\
\hline HCV RNA, $\times 10^{6}$, copies $/ \mathrm{mL}$ & $8.16(1.21)$ & $1.02(0.52)$ & $<.001$ \\
\hline $\mathrm{ALT}, \mathrm{IU} / \mathrm{L}$ & $97.94(8.24)$ & $64.28(8.07)$ & .005 \\
\hline ALT/ULN (n) & & & .273 \\
\hline$<1 x$ & 26 & 5 & \\
\hline$\geqq 1 x<2 x$ & 35 & 7 & \\
\hline$\geqq 2 x<3 x$ & 21 & 5 & \\
\hline$\geqq 3 x<4 x$ & 7 & 1 & \\
\hline$\geqq 4 x$ & 20 & 0 & \\
\hline ActiTest, A score & $0.51(0.03)$ & $0.51(0.06)$ & .928 \\
\hline Bilirubin, umol/L & $16.80(0.54)$ & $22.36(2.00)$ & $<.001$ \\
\hline $\mathrm{Cr}, \mathrm{umol} / \mathrm{L}$ & $71.80(4.34)$ & $65.42(4.35)$ & .301 \\
\hline INR & $1.02(0.01)$ & $1.12(0.03)$ & $<.001$ \\
\hline $\mathrm{Na}$, meq/L & $137.90(0.24)$ & $137.83(0.60)$ & .918 \\
\hline Platelet, $\times 10^{9} / \mathrm{L}$ & $176.61(5.67)$ & $121.44(9.73)$ & $<.001$ \\
\hline \multicolumn{4}{|l|}{ Child-Pugh grade $(n)$} \\
\hline A & & 16 & \\
\hline$B$ & & 2 & \\
\hline \multicolumn{4}{|l|}{$\mathrm{C}$} \\
\hline \multicolumn{4}{|l|}{ METAVIR F (n) } \\
\hline Fo & 0 & & \\
\hline F1 & 46 & & \\
\hline F2 & 40 & & \\
\hline F3 & 23 & & \\
\hline F4 & & 18 & \\
\hline METAVIR A (n) & & & .484 \\
\hline $\mathrm{A} 0$ & 31 & 3 & \\
\hline $\mathrm{A} 1$ & 60 & 10 & \\
\hline $\mathrm{A} 2$ & 16 & 5 & \\
\hline A3 & 2 & 0 & \\
\hline Hepatic steatosis (n) & & & 1.000 \\
\hline SO & 15 & 2 & \\
\hline $\mathrm{S1}$ & 37 & 6 & \\
\hline $\mathrm{S} 2$ & 52 & 10 & \\
\hline $\mathrm{S3}$ & 4 & 0 & \\
\hline S4 & 1 & 0 & \\
\hline
\end{tabular}


Table 1 Patient characteristics (Continued)

\begin{tabular}{llc}
\hline Liver SWW, m/s & $1.65(0.06)$ & $2.62(0.19)$ \\
\hline FibroTest, F score & $0.57(0.03)$ & $0.79(0.04)$ \\
\hline Continuous variables were presented as mean (standard error) and examined using the Student's $t$ test. Categorical variables were estimated using Fisher's exact \\
test. ALT, serum alanine aminotransferase; Cr, serum creatinine; INR, international normalized ratio of prothrombin time; METAVIR A, activity; METAVIR F, fibrosis; \\
Na, serum sodium; SWV, shear wave velocity.
\end{tabular}

were $0.196(P=.027)$ between ALT and METAVIR $\mathrm{F}$ stages; $0.305(P<.001)$ between ActiTest A scores and METAVIR F stages (Figure 1). Univariate linear regressions were both significant between ALT and ARFI LSM $\left(\mathrm{R}^{2}=0.074, P=.002\right)$, and between ActiTest A scores and ARFI LSM $\left(\mathrm{R}^{2}=0.198, P<.001\right)$ (Figure 2$)$.

\section{Histology}

The mean length of the included liver biopsy specimens was $21.7 \pm 3.3 \mathrm{~mm}$ (standard deviation, SD) (range, 15 to $32 \mathrm{~mm}$ ). Using the METAVIR F scoring system, 46 patients were staged as F1, 40 as F2, 23 as F3, and 18 as F4.

\section{ARFI LSM}

The ICC was 0.993 (95\% CI, 0.981-0.997; $P<.001$ ) for LSM. The descriptive statistics of the liver SWVs for the METAVIR stages were as follows: F1: mean, $1.283 \pm 0.037 \mathrm{~m} / \mathrm{s}$ (standard error of mean, SE); median, $1.205 \mathrm{~m} / \mathrm{s}$ (range, 0.96 to 2.21); F2: mean, $1.630 \pm 0.081 \mathrm{~m} / \mathrm{s} ;$ median, $1.590 \mathrm{~m} / \mathrm{s}$ (range, 0.98 to 3.28); F3: mean, $2.433 \pm 0.134 \mathrm{~m} / \mathrm{s}$; median, $2.395 \mathrm{~m} / \mathrm{s}$ (range, 1.09 to 3.58 ); F4: mean, $2.619 \pm 0.187 \mathrm{~m} / \mathrm{s}$; median, $2.525 \mathrm{~m} / \mathrm{s}$ (range, 1.06 to 4.56 ) (Figure 3).

The Spearman's rank correlation coefficient between liver SWV and METAVIR F stages was $0.696(P<.001)$. The overall intergroup difference in liver SWVs between METAVIR F1 to F4 was significant $(P<.001)$. Pair-wise comparisons also showed significant differences between groups: all $P<.001$, except $P=.412$ for F3 versus F4.

To classify METAVIR F stages, the areas under the ROC curves (AUCs) were ARFI LSM, 0.847 (95\% CI, 0.779-0.914) and FibroTest, 0.823 (95\% CI, 0.748-0.898), for F1 versus F2-4; ARFI LSM, 0.902 (95\% CI, 0.8350.970 ) and FibroTest, 0.812 (95\% CI, 0.735-0.888), for F1-2 versus F3-4; ARFI LSM, 0.831 (95\% CI, 0.7230.939) and FibroTest, 0.757 (95\% CI, 0.648-0.865), for F1-3 versus F4. A comparison of the AUCs [16] using liver SWV and FibroTest results showed insignificant differences between the following groups: F1 versus F2$4, P=.638 ; \mathrm{F} 1-2$ versus $\mathrm{F} 3-4, P=.086 ; \mathrm{F} 1-3$ versus $\mathrm{F} 4$, $P=.341$ (Figure 4).

The optimal cutoff values to classify METAVIR F stages using ARFI LSM were $1.55 \mathrm{~m} / \mathrm{s}$ for F1 versus F2-4 (74.1\% sensitivity, $87.0 \%$ specificity, $90.9 \%$ positive predictive value (PPV), 65.6\% negative predictive value (NPV), 78.8\% concordance, $21.2 \%$ discordance), $1.81 \mathrm{~m} / \mathrm{s}$ for F1-2 versus F3-4 (90.2\% sensitivity, $89.5 \%$ specificity, 80.4\% PPV, 95.0\% NPV, 89.7\% concordance, $10.3 \%$ discordance), and $1.98 \mathrm{~m} / \mathrm{s}$ for $\mathrm{F} 1-3$ versus $\mathrm{F} 4$ (88.9\% sensitivity, $79.8 \%$ specificity, $42.1 \%$ PPV, 97.8\% NPV, 81.1\% concordance, $18.9 \%$ discordance) (Table 2).
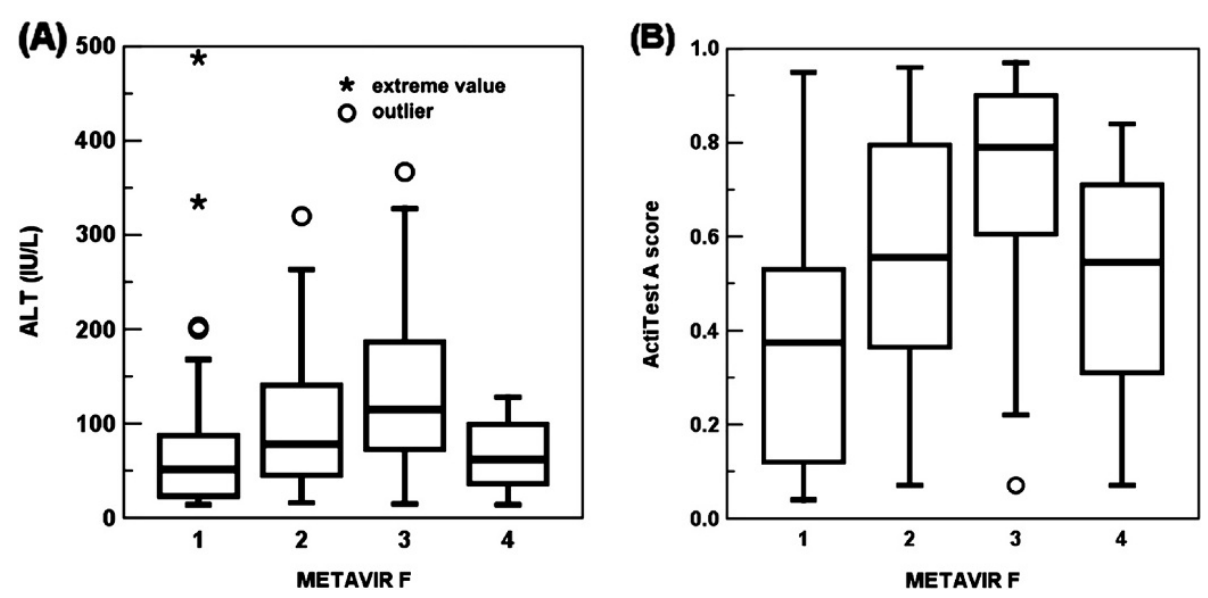

Figure 1 Box plot of necroinflammatory degrees corresponding to the METAVIR fibrosis (F) stages. The Spearman's rank correlation coefficients were $0.196(P=.027)$ between serum alanine aminotransferase (ALT) levels and METAVIR F stages $(\mathbf{A})$; $0.305(P<.001)$ between ActiTest A scores and METAVIR F stages (B). Pair-wise comparisons of ActiTest A scores showed significant differences between groups F1, F2, and F3: all $P<.005$. 

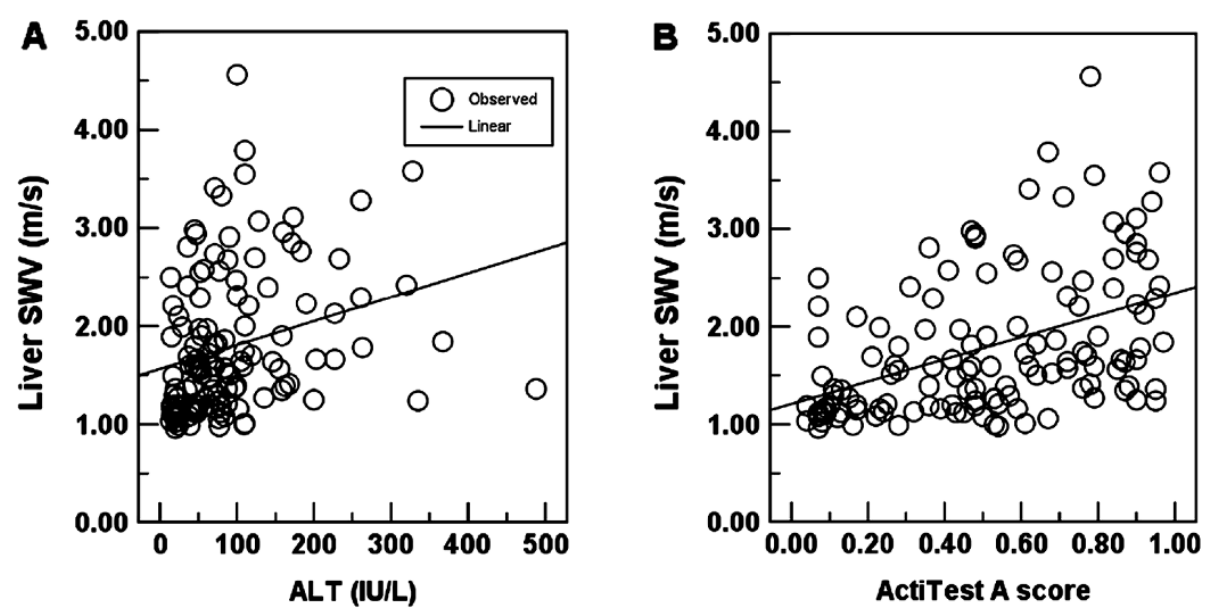

Figure 2 Univariate linear regressions between necroinflammatory degrees and liver shear wave velocity (SWV). Univariate linear regressions were both significant between serum alanine aminotransferase (ALT) levels and liver SWV $\left(R^{2}=0.074, P=.002\right)(\mathbf{A})$, and between ActiTest A scores and liver SWV $\left(R^{2}=0.198, P<.001\right)(B)$.

\section{Statistical analysis}

After excluding the HCV genotype $(P=.810)$, serum creatinine $(\mathrm{Cr})(P=.997)$, and sodium $(\mathrm{Na})(P=.388)$, univariate linear regression modelling selected BMI, HCV RNA, METAVIR F stages, ALT/ULN (40 IU/L) categories (model 1), ActiTest A scores (model 2) and METAVIR A grades (model 3), hepatic steatosis categories, bilirubin, INR, and platelet count (all $P<.25$ ), in addition to age

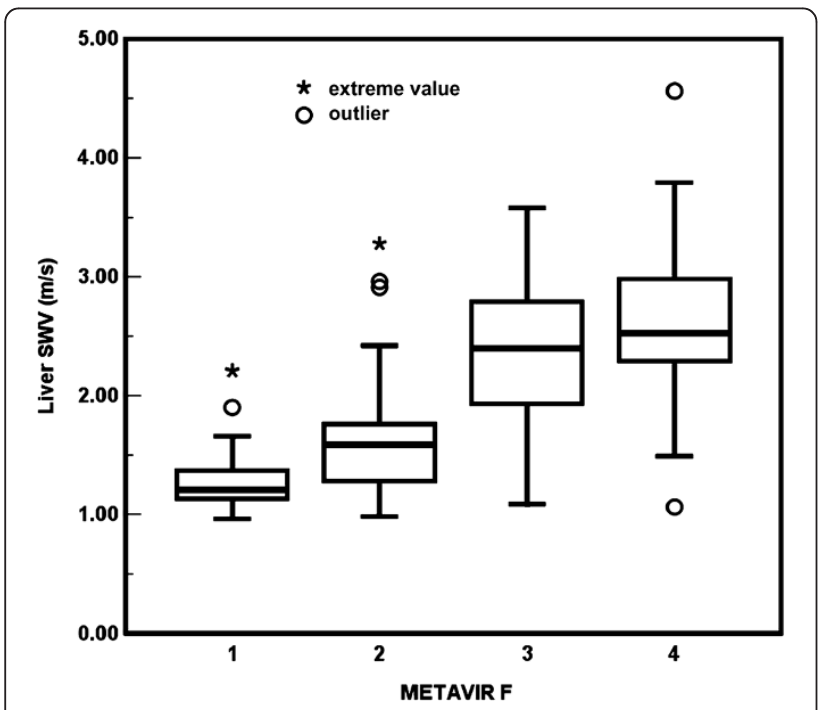

Figure 3 Box plot of liver stiffness corresponding to the METAVIR fibrosis (F) stages. The overall inter-group difference of liver shear wave velocity (SWV) among METAVIR F1 to F4 was significant $(P<.001)$. Pair-wise comparisons also showed significant differences between groups: all $P<.001$, except $P=.412$ for F3 versus F4.
$(P=.001)$ and sex $(P=.425)$, for the subsequent stepwise multiple linear regressions. The HCV RNA, hepatic steatosis categories, and serum bilirubin were excluded from the stepwise regressions for all three models.

Finally, in addition to BMI, METAVIR F stages, and platelet count, multiple linear regression models 1,2 , and 3 consistently identified serum ALT/ULN categories (model 1), ActiTest A scores (model 2) and METAVIR A grades (model 3) as significant independent explanatory factors for LSM (Table 3).

Thirty-two patients out of the 109 patients with METAVIR F1, F2 and F3 were false positive (32/109, 29\%). False positive case numbers were as follows: 8 (8/ 46, 17\%) (ARFI LSM $>1.55 \mathrm{~m} / \mathrm{s}$ ) in METAVIR F1; 8 (8/ $40,25 \%)(\mathrm{LSM}>1.81 \mathrm{~m} / \mathrm{s}) \quad$ in $\mathrm{F} 2 ; 16(16 / 23,70 \%)$ $(\mathrm{LSM}>1.98 \mathrm{~m} / \mathrm{s})$ in F3 stages.

Using ALT levels to discriminate the false positive $(n=32)$ from the non false positive $(n=77)$ of the 109 patients with METAVIR F1, F2 and F3, the AUC was 0.715 (SE, 0.053; 95\% CI, 0.612-0.819; $P<.001$ ). The ALT level of $109.5 \mathrm{IU} / \mathrm{L}$ was the optimal cutoff value with a sensitivity of $56.3 \%$ and a specificity of $81.8 \%$. Using the ActiTest A scores, the optimal cutoff was 0.35 (AUC, 0.736; SE, 0.051; 95\% CI, 0.636-0.835; $P<.001$; sensitivity, 93.8\%; specificity, 41.6\%)(Figure 5).

Using "ALT/ULN < $1 \mathrm{x}$ ", "METAVIR A0", and "ActiTest A score $0.00-0.35$ " as reference categories, univariate binary logistic regressions showed significant necroinflammatory effects on the false positivity of LSM to stage fibrosis in the 32 patients with METAVIR F1, F2 and F3. "ALT/ULN $\geqq 3 x$ " had an odds ratio (OR) of 15.0 (95\% CI, 2.9-76.6; $P=.001$ ). "METAVIR A2-3": OR, 32.7; 95\% CI; 6.4-166.5; $P<.001$. "ActiTest A score 0.36-0.75": OR, 

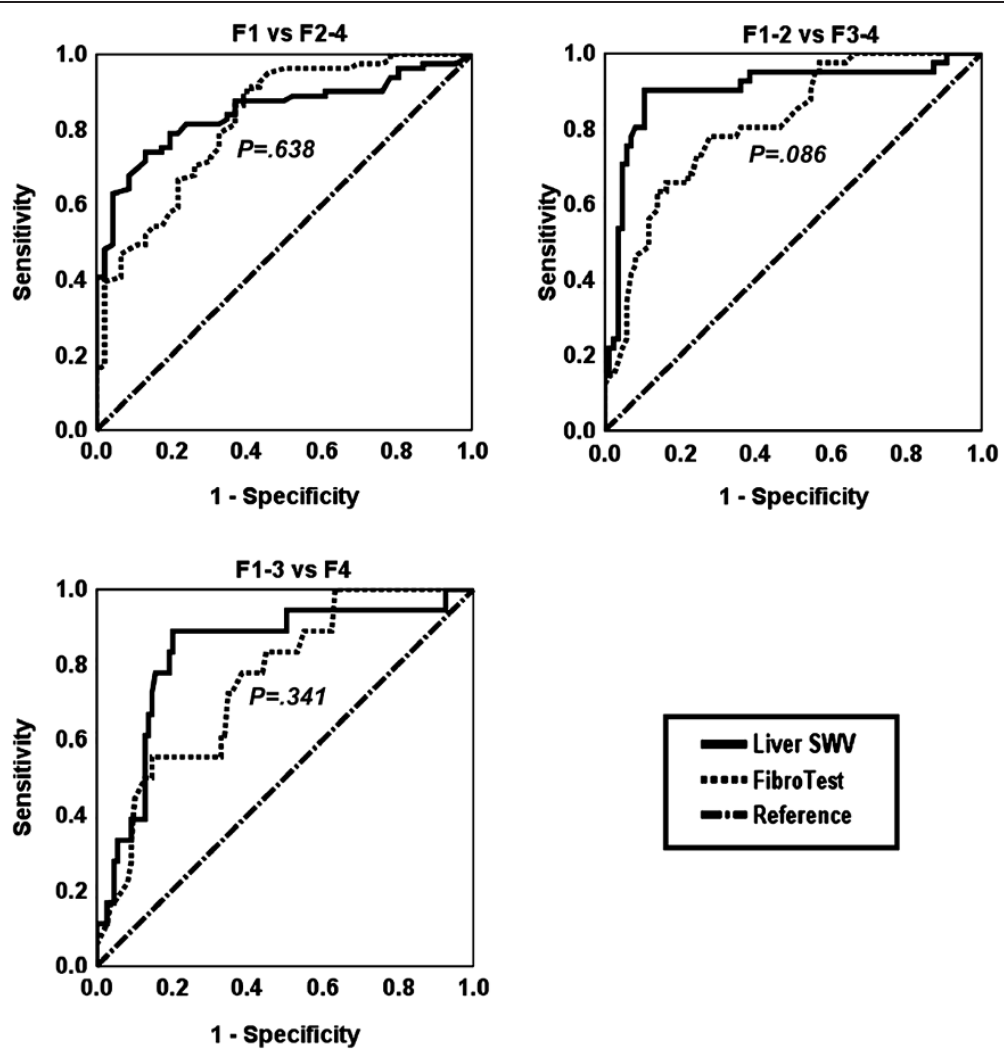

Figure 4 Receiver operating characteristic (ROC) curves to classify the METAVIR fibrosis stages. Two diagnostic modalities, liver shear wave velocity (SWV), and FibroTest (F score) were compared. $P$ value: significance for comparisons of areas under ROC curves between using liver SWV and FibroTest.

8.4; 95\% CI, 1.7-40.8; $P=.002$. "ActiTest A score 0.761.00”: OR, 11.9; 95\% CI, 2.5-57.2; $P=.002$ (Table 4).

\section{Discussion}

In the chronically injured liver, fibrogenesis is the complex dynamic interplay among various hepatic cell types and mediators in which the process of perpetuation follows initiation [4]. With the clinical application of magnetic resonance (MR) and ultrasound-based LSM, studies using MR elastography (MRE) [44], FibroScan, and ARFI elastography have demonstrated significant correlations between liver stiffness and liver fibrosis. However, liver stiffness and liver fibrosis are not equivalent.

In addition to the accumulation of the fibrotic extracellular matrix, other components of chronic liver disease, including cholestasis [31,32], cardiac congestion [33], and, particularly, the degree of necroinflammatory activity, can exaggerate ultrasound-based LSM. Patients with $\mathrm{CHC}$ usually manifest relatively stable serum ALT levels compared with the abrupt and fluctuating ALT levels in patients with chronic hepatitis B (CHB). Swelling of hepatocytes, interstitial edema, and infiltrates of inflammatory cells may increase liver stiffness in patients with acute hepatitis [45]. After adjusting for other demographic and biological covariates, the results of our study indicate that a $100 \mathrm{IU} / \mathrm{L}$ increase in serum ALT levels augmented liver stiffness values by approximately $0.155 \mathrm{~m} / \mathrm{s}$ (model $\mathrm{R}^{2}=0.630$, adjusted $\mathrm{R}^{2}=0.602$ ).

ActiTest is a biomarker of liver necroinflammatory histological activity validated in patients with $\mathrm{CHC}$. The accuracy of ActiTest for grading necroinflammatory activity in $\mathrm{HCV}$-infected patients was significantly higher than serum ALT alone [34]. In our study, the ActiTest A score model $\left(R^{2}=0.662\right.$, adjusted $\left.R^{2}=0.636\right)$ was superior to the models using serum ALT levels $\left(R^{2}=0.630\right.$, adjusted $\left.R^{2}=0.602\right)$, ALT/ULN categories $\left(R^{2}=0.661\right.$, adjusted $\left.R^{2}=0.629\right)$ and METAVIR A grades $\left(R^{2}=0.651\right.$, adjusted $\left.R^{2}=0.620\right)$ in explaining the LSM results.

The cutoffs of ARFI LSM in this study $(1.55 \mathrm{~m} / \mathrm{s}$ for $\mathrm{F} 1$ versus $\mathrm{F} 2-4 ; 1.81 \mathrm{~m} / \mathrm{s}$ for F1-2 versus F3-4) were in part higher than those in the latest study on $139 \mathrm{CHC}$ cases by Rizzo et al. $(1.3 \mathrm{~m} / \mathrm{s}$ for F1 versus F2-4; $1.7 \mathrm{~m} / \mathrm{s}$ for F1-2 versus F3-4)[12]. Necroinflammatory effects explain most of the differences. First, the overall necroinflammatory degree was higher (Student's $t$-test, $P=.034$ ) in this study (mean ALT, 93.2; SE, 7.2 IU/L; $\mathrm{n}=127$ ) than for Rizzo et al. (mean ALT, 77.2; SD, 33.0 IU/L; 
Table 2 The diagnostic performance of liver SWV and FibroTest in classifying METAVIR fibrosis (F) stages

\begin{tabular}{lccc}
\hline & \multicolumn{3}{c}{ METAVIR } \\
\cline { 2 - 4 } & F1 vs F2-4 & F1-2 vs F3-4 & F1-3 vs F4 \\
\hline AUC (95\% Cl) & $0.847(0.779-0.914)$ & $0.902(0.835-0.970)$ & 0.831 \\
SE of AUC & 0.034 & $0.723-0.939)$ \\
Cutoff (m/s) & 1.55 & 1.81 & 0.055 \\
Sensitivity & $74.1 \%$ & $90.2 \%$ & 1.98 \\
Specificity & $87.0 \%$ & $89.5 \%$ & $88.9 \%$ \\
PPV & $90.9 \%$ & $80.4 \%$ & $79.8 \%$ \\
NPV & $65.6 \%$ & $95.0 \%$ & $42.1 \%$ \\
Concordance & $78.8 \%$ & $89.7 \%$ & $97.8 \%$ \\
Discordance & $21.2 \%$ & $10.3 \%$ & $81.1 \%$ \\
+LR & 5.7 & 8.6 & $18.9 \%$ \\
-LR & 0.3 & 0.1 & 4.4 \\
DOR(95\% Cl) & $19.0(7.1-51.3)$ & $79.1(22.9-273.8)$ & $31.6(6.8-147.9)$ \\
& FibroTest & & \\
AUC (95\% Cl) & $0.823(0.748-0.898)$ & $0.812(0.735-0.888)$ & $0.757(0.648-0.865)$ \\
SE of AUC & 0.038 & 0.039 & 0.055 \\
\hline Concordance & & &
\end{tabular}

Concordance $=($ true positive $\mathrm{n}+$ true negative $\mathrm{n}) / 127$, and discordance $=($ false positive $\mathrm{n}+$ false negative $\mathrm{n}) / 127$; AUC, area under the receiver operating characteristic curve; DOR, diagnostic odds ratio; LR, likelihood ratio; NPV, negative predictive value; PPV, positive predictive value; SE, standard error; SWV, shear wave velocity.

$\mathrm{n}=139)$. Second, the distribution of necroinflammation tended to be more severe in METAVIR F3 and F2 than F1 stages in this study (Figure 1). Thus, the LSM values were more augmented in F3 and F2 than F1 stages. The analyses of false positivity in patients with F1 to F3 also correlated well with the necroinflammatory effects (Table 4). Third, cirrhotic Taiwanese patients (mean age, 62.7; SE, 1.5 years; $\mathrm{n}=18$ ) referred to the unit for SOC tended to be older (Student's $t$-test, $P=.0083$ ) than those in the study by Rizzo et al. (mean age, 55; SD, 12 years; $\mathrm{n}=139)$ [12]. Despite the advanced countrywide education and health insurance coverage, these patients were characterized by poor patient compliance and late diagnosis. Although age did not affect LSM significantly, as demonstrated in models in the present and previous studies, an older age may have resulted in significant fibrosis progression in these cirrhotic Taiwanese patients despite the relatively compensated liver reserves.

Despite the variation among previous investigations, several recent studies have demonstrated the necroinflammatory effects on liver stiffness by evolving analyses.

In a longitudinal study using FibroScan, Sagir et al. [21] observed false positivity for cirrhosis (cutoff $>12.5$ $\mathrm{kPa})$ in 15 of 20 non-cirrhotic patients with acute liver damage of various etiologies. In 6 patients, the LSMs dropped below $12.5 \mathrm{kPa}$ with normalized ALT levels during follow-up. Using a longitudinal analysis, Arena et al. [20] demonstrated significant correlations between sequential serum ALT levels and LSM results at different time points. Although these studies showed the need for caution when analyzing LSM in patients with necroinflammatory flares, they did not include regression estimates. Seo et al. [25] demonstrated that peak ALT levels significantly explained peak LSMs in 31 patients in acute hepatitis A via linear regressions adjusting for age and sex.

A cross-sectional study by Le et al. [23] showed that LSM using FibroScan in 158 patients was independently associated with histological necroinflammatory grading, but irrespective of serum ALT levels. Fung et al. [26] reported a suboptimal PPV (as low as 10\%) for LSM using FibroScan to diagnose true cirrhosis in 102 patients (median age, 41; range, 18-63 years) with active hepatitis B (median serum ALT, 89; range, 46-501 IU/ L). Multiple logistic regressions by Myers et al. [29] showed that serum ALT levels greater than the optimal cutoff value $60 \mathrm{IU} / \mathrm{L}$ from ROC analysis were significantly correlated with the discordance (at least 2 stages between FibroScan and biopsy). Chan et al. [24] and

Table 3 Three multiple linear regression models to identify independent significant factors that explain liver stiffness

\begin{tabular}{|c|c|c|c|c|c|c|c|c|c|}
\hline \multirow[t]{2}{*}{ Variable } & \multicolumn{3}{|c|}{ Model 1} & \multicolumn{3}{|c|}{ Model 2} & \multicolumn{3}{|c|}{ Model 3} \\
\hline & B & SE & $P$ & B & SE & $P$ & B & SE & $P$ \\
\hline Age, year & -.003 & .004 & .512 & -.004 & .004 & .353 & -.004 & .004 & .366 \\
\hline Male gender & -.193 & .084 & .024 & -.194 & .083 & .020 & -.124 & .085 & .147 \\
\hline BMI, $\mathrm{kg} / \mathrm{m}^{2}$ & .032 & .013 & .013 & .036 & .012 & .005 & .026 & .012 & .041 \\
\hline INR & .972 & .572 & .092 & .890 & .564 & .117 & 1.146 & .573 & .048 \\
\hline Platelet, $\times 10^{9} / \mathrm{L}$ & -.003 & .001 & .002 & -.003 & .001 & .003 & -.002 & .001 & .007 \\
\hline METAVIR & & & & & & & & & \\
\hline 52 & .089 & .113 & .430 & .049 & .113 & .666 & .123 & .114 & .284 \\
\hline F3 & .739 & .154 & $<.001$ & .670 & .155 & $<.001$ & .670 & .161 & $<.001$ \\
\hline 51 & .989 & .168 & $<.001$ & .954 & .165 & $<.001$ & .892 & .171 & $<.001$ \\
\hline
\end{tabular}

ALT/ULN

$\geqq 1<2 x \quad 307 \quad 110 \quad .006$

$\geqq \mathbf{2}<\mathbf{3 x} \quad .429 .122 \quad .001$

$\geqq 3 \quad .523 \quad .133<.001$

ActiTest A score $\quad .717 \quad .163<.001$

\section{METAVIR A}

A1

$\begin{array}{lll}189 & .060 & .061\end{array}$

A2-3

$.551 .144<.001$

$\begin{array}{llll}\mathrm{R}^{2} & .661 & .662 & .651\end{array}$

$\begin{array}{llll}\text { adjusted } R^{2} & .629 & .636 & .620\end{array}$

Variable references: female gender, METAVIR F1, ALT/ULN <1x, METAVIR A0; ALT, serum alanine aminotransferase; $B$, coefficient; INR, international normalized ratio of prothrombin time; SE, standard error of coefficient; ULN, upper limit of normal. 

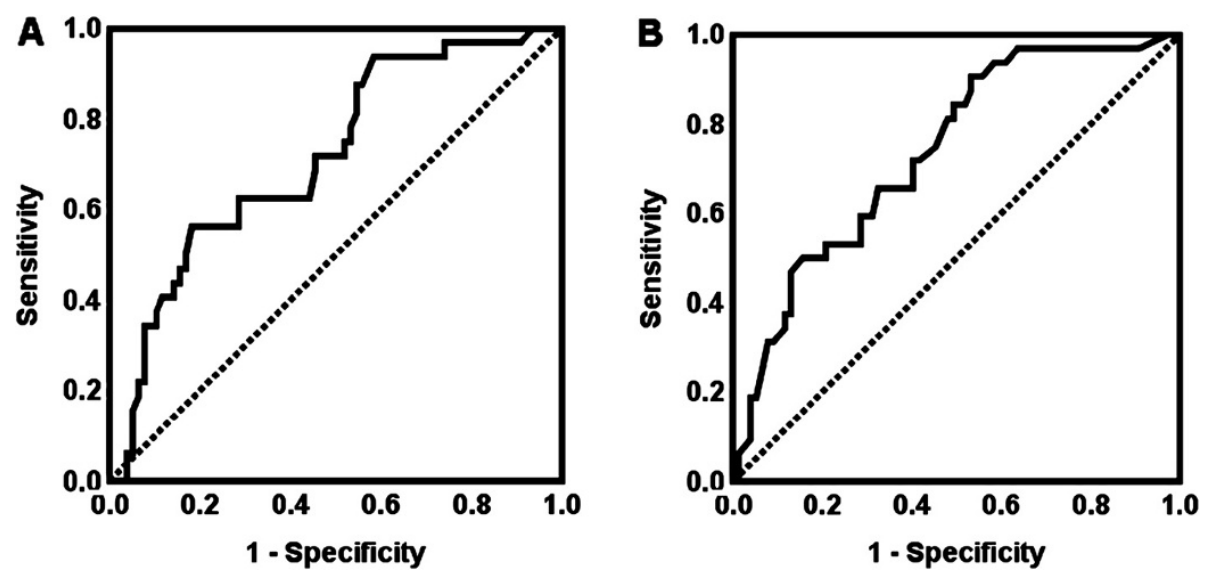

Figure 5 Receiver operating characteristic (ROC) curves to classify the false positivity in METAVIR F1 to F3. Using alanine aminotransferase (ALT) levels (A) to discriminate the false positive in liver fibrosis evaluation using acoustic radiation force impulse elastography $(n=32)$ from the non false positive $(n=77)$ of the 109 patients with METAVIR F1, F2 and F3, the area under ROC curve (AUC) was 0.715 (standard error, SE, 0.053; 95\% Cl, 0.612-0.819; $P<.001)$. The ALT level of $109.5 \mathrm{IU} / \mathrm{L}$ was the optimal cutoff value with a sensitivity of $56.3 \%$ and a specificity of $81.8 \%$. Using the ActiTest A scores (B), the optimal cutoff was 0.35 (AUC, 0.736; SE, 0.051; 95\% Cl, 0.636-0.835; $P<.001$; sensitivity, 93.8\%; specificity, 41.6\%)

Kim et al. [27] proposed distinct sets of cutoff values stratified by distinct ALT profiles.

Tapper et al. [30] further delineated the positive necroinflammatory effects on LSM using FibroScan through linear regressions in $684 \mathrm{HCV}$ patients with METAVIR F0, F1 and F2. Logistic regressions also showed that false positivity of liver fibrosis staging was associated with both histological and serum hepatic necroinflammatory activity. Using $14.5 \mathrm{kPa}$ as the cutoff of cirrhosis, grade 3 inflammation had an OR of 9.10 (95\% CI, 2.49-33.4). Likewise, serum levels of ALT greater than $80 \mathrm{IU} / \mathrm{L}$ and $120 \mathrm{IU} / \mathrm{L}$ had ORs of 3.84 (95\% CI, 2.10-7.00) and 4.10 (95\% CI, 2.18-7.69) over references, respectively. Yoon et al. [15] used ARFI elastography to demonstrate a significant correlation (Pearson's $r=0.431, P<.05)$ between LSM values and serum ALT levels, and a marked positive effect of histological necroinflammatory activity on LSM. However, this study did not adjust for other relevant essential covariates. ARFI elastography performed potentially better for patients with normal ALT than for those with high ALT (AUCs, 0.88 versus 0.73 for METAVIR F1-2 versus F3-4, 0.92 versus 0.72 for F1-3 versus F4). However, AUCs were not statistically compared. The cutoff values tended to be lower for patients with normal ALT than for those with high ALT levels (ALT $>40$ IU/L) $(1.09$ versus $1.16 \mathrm{~m} / \mathrm{s}$ for F1-2 versus F3-4, and 1.81 versus $2.23 \mathrm{~m} / \mathrm{s}$ for F1-3 versus F4, respectively).

In contrast to results indicating positive necroinflammatory effects on liver stiffness, Harata et al. [32] identified a negative correlation between serum ALT levels and liver stiffness in patients with cholestasis. Cholestasis is a condition in which the release of hydrostatic pressure with synchronous necroinflammatory activity can, paradoxically, reduce the values of LSM using FibroScan. Rizzo et al. [12] found distinct necroinflammatory effects on LSM between using FibroScan and ARFI elastography. Colombo et al. [13] demonstrated an insignificant (Spearman's rank) correlation between necroinflammation and LSM. The serum ALT specific cutoffs of $202 \mathrm{CHB}$ patients in a study by Cardoso et al. did not increase the diagnostic performances using FibroScan for liver fibrosis evaluation [28].

Although hepatic steatosis is prevalent in patients with $\mathrm{CHC}$, the linear regression analysis in this study did not identify steatosis as a significant independent explanatory factor for the ARFI LSM results. However, larger sample sizes are required to further delineate the effects of more severe forms of steatosis (S3, S4) on ARFI LSM results. Motosugi et al. [46] also demonstrated the insignificance of different ARFI LSM results among four grades of steatosis $(P=.9018)$. Using MRE in a mouse model, Yin et al. [47] showed that steatosis did not correlate significantly with liver stiffness at each liver fibrosis stage.

This study shows that BMI significantly and independently explain the results of ARFI LSM (Table 3). Similarly, Roulot et al. [48] used FibroScan to show that liver stiffness was significantly higher $(P=.0005)$ in obese patients (with $\mathrm{BMI}>30 \mathrm{~kg} / \mathrm{m}^{2}$ ) than in overweight or normal patients, after adjusting for age, sex, ALT, aspartate aminotransferase (AST) and ferritin. Baba et al. also demonstrated a significant association between BMI and liver stiffness using FibroScan. Hepatic steatosis, however, was not evaluated in adjusted models [49]. Conversely, Talwalkar et al. [50] reported an insignificant (linear) correlation between BMI and LSM results using MRE. 
Table 4 Factors associated with false positivity in patients with METAVIR F1, F2 and F3

\begin{tabular}{|c|c|c|c|c|}
\hline \multirow[t]{2}{*}{ Variable } & \multirow{2}{*}{$\begin{array}{c}\text { Non FP } \\
n=77\end{array}$} & \multirow{2}{*}{$\begin{array}{c}F P \\
n=32\end{array}$} & \multirow[t]{2}{*}{ OR(95\% Cl) } & \multirow[t]{2}{*}{$P$ value } \\
\hline & & & & \\
\hline Age, year & $50.8(1.4)$ & $53.4(2.0)$ & & .316 \\
\hline Gender, male/female (n) & $35 / 42$ & $16 / 16$ & & .679 \\
\hline $\mathrm{BMl}, \mathrm{kg} / \mathrm{m}^{2}$ & $24.34(0.37)$ & $25.37(0.68)$ & & .155 \\
\hline HCV genotype, 1/non-1 (n) & $38 / 39$ & $15 / 17$ & & .836 \\
\hline HCV RNA, x $10^{6}$, copies $/ \mathrm{mL}$ & $7.14(1.12)$ & $10.61(3.12)$ & & .194 \\
\hline ALT, IU/L & $83.01(9.40)$ & 133.88(15.02) & & .004 \\
\hline \multicolumn{5}{|l|}{ ALT/ULN (n) } \\
\hline$<1 x$ & 24 & 2 & reference & \\
\hline$\geqq 1 x<2 x$ & 25 & 10 & $4.8(0.9-24.2)$ & .057 \\
\hline$\geqq 2 x<3 x$ & 16 & 5 & $3.8(0.6-21.7)$ & .140 \\
\hline$\geqq 3 x$ & 12 & 15 & 15.0(2.9-76.6) & $.001^{*}$ \\
\hline ActiTest A score (n) & & & & . \\
\hline $0.00-0.35$ & 31 & 2 & reference & \\
\hline $0.36-0.75$ & 24 & 13 & $8.4(1.7-40.8)$ & $.008^{*}$ \\
\hline $0.76-1.00$ & 22 & 17 & $11.9(2.5-57.2)$ & $.002^{*}$ \\
\hline Bilirubin, umol/L & $15.76(0.56)$ & 19.30(1.12) & & .002 \\
\hline $\mathrm{Cr}, \mathrm{umol} / \mathrm{L}$ & $70.82(2.82)$ & $74.14(4.55)$ & & .817 \\
\hline INR & $1.00(0.01)$ & $1.05(0.02)$ & & .007 \\
\hline $\mathrm{Na}$, meq/L & $137.94(0.28)$ & $137.81(0.46)$ & & .527 \\
\hline Platelet, $\times 10^{9} / \mathrm{L}$ & $184.21(6.20)$ & $158.31(9.37)$ & & .025 \\
\hline METAVIR F1/2/3 (n) & $38 / 32 / 7$ & $8 / 8 / 16$ & & $<.001$ \\
\hline \multicolumn{5}{|l|}{ METAVIR A (n) } \\
\hline $\mathrm{AO}$ & 28 & 3 & reference & \\
\hline $\mathrm{A} 1$ & 45 & 15 & $3.1(0.8-11.7)$ & .094 \\
\hline $\mathrm{A} 2-3$ & 4 & 14 & $32.7(6.4-166.5)$ & $<.001^{*}$ \\
\hline Hepatic steatosis S0/1/2/3/4(n) & $10 / 28 / 35 / 3 / 1$ & $5 / 9 / 17 / 1 / 0$ & & .903 \\
\hline Liver SWV, m/s & $1.34(0.03)$ & $2.40(0.11)$ & & $<.001$ \\
\hline FibroTest, F score & $0.51(0.03)$ & $0.72(0.04)$ & & $<.001$ \\
\hline
\end{tabular}

Similar to the report by Talwalkar et al. [50], the effects of age and sex on ARFI LSM were insignificant in this study. Previously demonstrated as a significant factor explaining liver fibrosis evaluation [51], platelet count was strongly associated with ARFI LSM after adjustment, unlike the inconsistency and insignificance of INR (Table 3).

To minimize histology bias [6,52], the percutaneous liver biopsy in this study immediately followed ARFI LSM. Specimens were of adequate length, and an expert pathologist interpreted histological findings. Unreliable cases or failed ARFI measurements were primarily derived from obese patients with poor transient apnea maneuvers. Thus, future studies may analyze factors associated with unreliable cases or failure measurements. The present analyses show that the $\mathrm{R}^{2}$ values were modest for the regression models; therefore, other potential explanatory factors, especially direct tissue markers [45], must be identified to construct an optimal explanatory model (Table 3).

Future analyses would require a larger sample size to develop cutoffs and perform validations that are potentially more reliable and stable than those of this study. These investigations may compare the diagnostic performances of ARFI LSM with another promising diagnostic modality: MRE. Although FibroTest was initially proposed as a similar first-line approach to histology for prediction of 5-year 
survival in patients with $\mathrm{CHC}[37,38]$, it may also serve as a standard of reference, in addition to METAVIR F staging, for evaluating the diagnostic value of ARFI LSM in baseline or chronological analysis.

The limitations of this study include the lack of stratification of patients with cirrhosis into compensated and decompensated (excluded) groups. The absence of a decompensated group, which may have a high and broad spectrum for LSM, may have caused the insignificant differences in LSM results between METAVIR F3 and F4 $(P=.412)$ before adjusting for other covariates. Further analyses should identify optimal cutoffs for LSM to stratify the broad cirrhosis category and enable risk estimation of end points in chronological analyses [53]. This study does not identify the cutoff for LSM between METAVIR F0 and F1-F4 because of the lack of wellestablished criteria for recruiting true cases with F0 fibrosis without liver biopsy.

Future noninvasive liver fibrosis evaluation tools should focus on exact staging of fibrosis, rather than classifying patients into categories of milder versus more severe fibrosis stages. In the milder strata of liver fibrosis, necroinflammation can easily overwhelm the fibrotic effects on LSM [30]. In this study, the diagnostic performance of ARFI LSM was potentially limited when distinguishing between METAVIR F1 and F2 categories too. The insignificant regression coefficient in Table 3 reflects the potentially limited sensitivity for ARFI LSM in distinguishing F2 from F1. Therefore, further research is needed prior to the clinical application of ARFI LSM for surveillance of progression, or regression, of liver fibrosis. The real clinical relevance between METAVIR F1 and F2 is still not known with respect to either prognosis or risk of disease progression over the short to intermediate term of 5 to 10 years [5]. Future studies should develop algorithms based on a combination of ARFI LSM and essential serum markers for liver fibrosis evaluation to minimize false positivity of fibrosis staging $[24,27,54]$. A further limitation is that this study did not perform standardization of AUCs based on the prevalence of liver fibrosis stages [55].

\section{Conclusions}

In conclusion, the degree of concurrent hepatic necroinflammatory activity independently and significantly exaggerated liver fibrosis evaluation using ARFI LSM. However, comparisons of concurrent FibroTest and ARFI LSM indicate that ARFI LSM may provide a promising alternative, or adjunctive predictive solution, for evaluating liver fibrosis in patients with $\mathrm{CHC}$.

\section{Abbreviations}

ALD: Alcoholic Liver Disease; ALT: ALanine aminoTransferase; AST: ASpartate aminoTransferase; ARFI: Acoustic Radiation Force Impulse; AUC: Area Under the receiver operating characteristic Curve; BMI: Body Mass Index;
CHB: Chronic Hepatitis B; CHC: Chronic Hepatitis C; Cl: Confidence Interval; Cr: Creatinine; DOR: Diagnostic Odds Ratio; FP: False Positive; GGT: $\gamma$-Glutamyl Transpeptidase; HBV: Hepatitis B Virus; HCV: Hepatitis C Virus;

HCC: HepatoCellular Carcinoma; HIV: Human Immunodeficiency Virus; ICC: Intraclass Correlation Coefficient; INR: International Normalized Ratio; IQR: InterQuartile Range; +LR: Positive Likelihood Ratio; -LR: Negative Likelihood Ratio; LSM: Liver Stiffness Measurement; METAVIR A: Activity; METAVIR F: Fibrosis; MRE: Magnetic Resonance Elastography; Na: Sodium; NPV: Negative Predictive Value; PPV: Positive Predictive Value; ROC: Receiver Operating Characteristic; ROI: Region Of Interest; SD: Standard Deviation; SE: Standard Error of mean; SOC: Standard Of Care; SWV: Shear Wave Velocity; ULN: Upper Limit of Normal; VTQ: Virtual Touch Quantification.

\section{Competing interests}

The authors declare that they have no competing interests.

\section{Authors' contributions}

SHC, YFL and CYP designed the study and analyzed the data. SHC performed the ARFI LSMS. IPC performed the histological interpretations. SHC, YFL, HCL, JTK, CYP, PHC, WPS, and IPC participated in the drafting of the manuscript. All of the authors read and approved the final manuscript.

\section{Acknowledgments}

This study was supported, in part, by the Department of Medical Research, China Medical University Hospital (grant DMR-100-017).

\section{Author details}

'Division of Hepatogastroenterology, Department of Internal Medicine, China Medical University Hospital, No 2 Yuh-Der Road, Taichung 40402, Taiwan.

${ }^{2}$ China Medical University, Taichung 40402, Taiwan. ${ }^{3}$ Institute of Biostatistics Center, China Medical University, Taichung 40402, Taiwan. ${ }^{4}$ College of Chinese Medicine, China Medical University, Taichung 40402, Taiwan. ${ }^{5}$ School of Medicine, China Medical University, Taichung 40402, Taiwan. ${ }^{6}$ Department of Pathology, China Medical University Hospital, Taichung 40402, Taiwan.

Received: 24 February 2012 Accepted: 2 August 2012

Published: 10 August 2012

\section{References}

1. Yu ML, Chuang WL: Treatment of chronic hepatitis C in Asia: when East meets West. J Gastroenterol Hepatol 2009, 24:336-345.

2. Bizollon T, Ahmed SN, Radenne S, Chevallier M, Chevallier P, Parvaz P, Guichard S, Ducerf C, Baulieux J, Zoulim F, Trepo C: Long term histological improvement and clearance of intrahepatic hepatitis $C$ virus RNA following sustained response to interferon-ribavirin combination therapy in liver transplanted patients with hepatitis C virus recurrence. Gut 2003, 52:283-287.

3. Wang $\mathrm{JH}$, Changchien $\mathrm{CS}$, Hung $\mathrm{CH}$, Tung WC, Kee KM, Chen $\mathrm{CH}$, Hu TH, Lee CM, Lu SN: Liver stiffness decrease after effective antiviral therapy in patients with chronic hepatitis C: longitudinal study using FibroScan. J Gastroenterol Hepatol 2010, 25:964-969.

4. Moreira RK: Hepatic stellate cells and liver fibrosis. Arch Pathol Lab Med 2007, 131:1728-1734.

5. Lai $\mathrm{M}$, Afdhal $\mathrm{NH}$ : Editorial: staging liver fibrosis in hepatitis $\mathrm{C}$ : a challenge for this decade. Am J Gastroenterol 2011, 106:2121-2122.

6. Regev A, Berho M, Jeffers LI, Milikowski C, Molina EG, Pyrsopoulos NT, Feng ZZ, Reddy KR, Schiff ER: Sampling error and intraobserver variation in liver biopsy in patients with chronic HCV infection. Am J Gastroenterol 2002, 97:2614-2618.

7. Friedrich-Rust M, Nierhoff J, Lupsor M, Sporea I, Fierbinteanu-Braticevici C, Strobel D, Takahashi H, Yoneda M, Suda T, Zeuzem S, Herrmann E: Performance of Acoustic Radiation Force Impulse imaging for the staging of liver fibrosis: a pooled meta-analysis. J Viral Hepat 2012, 19: e212-e219.

8. Friedrich-Rust M, Wunder K, Kriener S, Sotoudeh F, Richter S, Bojunga J, Herrmann E, Poynard T, Dietrich CF, Vermehren J, Zeuzem S, Sarrazin C: Liver fibrosis in viral hepatitis: noninvasive assessment with acoustic radiation force impulse imaging versus transient elastography. Radiology 2009, 252:595-604.

9. Fierbinteanu-Braticevici C, Andronescu D, Usvat R, Cretoiu D, Baicus C, Marinoschi G: Acoustic radiation force imaging sonoelastography for 
noninvasive staging of liver fibrosis. World J Gastroenterol 2009, 15:5525-5532

10. Lupsor M, Badea R, Stefanescu H, Sparchez Z, Branda H, Serban A, Maniu A: Performance of a new elastographic method (ARFI technology) compared to unidimensional transient elastography in the noninvasive assessment of chronic hepatitis C Preliminary results. J Gastrointestin Liver Dis 2009, 18:303-310.

11. Takahashi $H$, Ono N, Equchi $Y$, Equchi T, Kitajima $Y$, Kawaguchi $Y$, Nakashita S, Ozaki I, Mizuta T, Toda S, Kudo S, Miyoshi A, Miyazaki K, Fujimoto K: Evaluation of acoustic radiation force impulse elastography for fibrosis staging of chronic liver disease: a pilot study. Liver Int 2010, 30:538-545.

12. Rizzo L, Calvaruso V, Cacopardo B, Alessi N, Attanasio M, Petta S, Fatuzzo F, Montineri A, Mazzola A, L'abbate L, Nunnari G, Bronte F, Di Marco V, Craxì A Cammà C: Comparison of transient elastography and acoustic radiation force impulse for non-invasive staging of liver fibrosis in patients with chronic hepatitis C. Am J Gastroenterol 2011, 106:2112-2120.

13. Colombo S, Buonocore M, Del Poggio A, Jamoletti C, Elia S, Mattiello M, Zabbialini D, Del Poggio P: Head-to-head comparison of transient elastography (TE), real time tissue elastography (RTE), and acoustic radiation force impulse (ARFI) imaging in the diagnosis of liver fibrosis. J Gastroenterol 2012, 47:461-469.

14. Sporea I, Sirli RL, Deleanu A, Popescu A, Focsa M, Danila M, Tudora A: Acoustic radiation force impulse elastography as compared to transient elastography and liver biopsy in patients with chronic hepatopathies. Ultraschall Med 2011, 32:S46-S52.

15. Yoon KT, Lim SM, Park JY, Kim DY, Ahn SH, Han KH, Chon CY, Cho M, Lee JW, Kim SU: Liver stiffness measurement using acoustic radiation force impulse (ARFI) elastography and effect of necroinflammation. Dig Dis Sci 2012, 57:1682-1691.

16. DeLong ER, DeLong DM, Clarke-Pearson DL: Comparing the areas under two or more correlated receiver operating characteristic curves: a nonparametric approach. Biometrics 1988, 44:837-845.

17. Toshima T, Shirabe K, Takeishi K, Motomura T, Mano Y, Uchiyama H, Yoshizumi T, Soejima Y, Taketomi A, Maehara Y: New method for assessing liver fibrosis based on acoustic radiation force impulse: a special reference to the difference between right and left liver. J Gastroenterol 2011, 46:705-711.

18. Goertz RS, Zopf Y, Jugl V, Heide R, Janson C, Strobel D, Bernatik T, Haendl T: Measurement of liver elasticity with acoustic radiation force impulse (ARFI) technology: an alternative noninvasive method for staging liver fibrosis in viral hepatitis. Ultraschall Med 2010, 31:151-155

19. Bota S, Sporea I, Sirli R, Popescu A, Dănilă M, Sendroiu M: Factors that influence the correlation of acoustic radiation force impulse (ARFI), elastography with liver fibrosis. Med Ultrason 2011, 13:135-140.

20. Arena U, Vizzutti F, Corti G, Ambu S, Stasi C, Bresci S, Moscarella S, Boddi V, Petrarca A, Laffi G, Marra F, Pinzani M: Acute viral hepatitis increases liver stiffness values measured by transient elastography. Hepatology 2008, 47:380-384.

21. Sagir A, Erhardt A, Schmitt M, Häussinger D: Transient elastography is unreliable for detection of cirrhosis in patients with acute liver damage. Hepatology 2008, 47:592-595.

22. Coco B, Oliveri F, Maina AM, Ciccorossi P, Sacco R, Colombatto P, Bonino F, Brunetto MR: Transient elastography: a new surrogate marker of liver fibrosis influenced by major changes of transaminases. J Viral Hepat 2007 14:360-369.

23. Lee da M, Moon EJ, Hwang JA, Lee MS, Cheong JY, Cho SW, Kim YB, Kim DJ, Hwang SG, Yang JM: Factors associated with liver stiffness in chronic liver disease. Korean J Hepatol 2009, 15:464-473.

24. Chan HL, Wong GL, Choi PC, Chan AW, Chim AM, Yiu KK, Chan FK, Sung JJ, Wong WW: Alanine aminotransferase-based algorithms of liver stiffness measurement by transient elastography (Fibroscan) for liver fibrosis in chronic hepatitis B. J Viral Hepat 2009, 16:36-44.

25. Seo $Y S$, Lee $K G$, Jung ES, An H, Park S, Keum B, Yim HJ, Jeen $Y T$, Chun HJ, Kim CD, Ryu HS, Um SH: Dynamic changes in liver stiffness during the course of acute hepatitis A. Scand J Gastroenterol 2010, 45:449-456.

26. Fung J, Lai CL, Chan SC, But D, Seto WK, Cheng C, Wong DK, Lo CM, Fan ST, Yuen MF: Correlation of liver stiffness and histological features in healthy persons and in patients with occult hepatitis $B$, chronic active hepatitis B, or hepatitis B cirrhosis. Am J Gastroenterol 2010, 105:1116-1122.
27. Kim BK, Han KH, Park JY, Ahn SH, Chon CY, Kim JK, Paik YH, Lee KS, Park YN, Kim do Y: A novel liver stiffness measurement-based prediction model for cirrhosis in hepatitis B patients. Liver Int 2010, 30:1073-1081.

28. Cardoso AC, Carvalho-Filho RJ, Stern C, Dipumpo A, Giuily N, Ripault MP, Asselah T, Boyer N, Lada O, Castelnau C, Martinot-Peignoux M, Valla DC, Bedossa P, Marcellin P: Direct comparison of diagnostic performance of transient elastography in patients with chronic hepatitis $B$ and chronic hepatitis C. Liver Int 2012, 32:612-621.

29. Myers RP, Pomier-Layrargues G, Kirsch R, Pollett A, Beaton M, Levstik M, Duarte-Rojo A, Wong D, Crotty P, Elkashab M: Discordance in fibrosis staging between liver biopsy and transient elastography using the FibroScan XL probe. J Hepatol 2012, 56:564-570.

30. Tapper EB, Cohen EB, Patel K, Bacon B, Gordon S, Lawitz E, Nelson D, Nasser IA, Challies T, Afdhal N: Levels of alanine aminotransferase confound use of transient elastography to diagnose fibrosis in patients with chronic HCV infection. Clin Gastroenterol Hepatol 2012, (). Epub ahead of print.

31. Millonig G, Reimann FM, Friedrich S, Fonouni H, Mehrabi A, Büchler MW, Seitz HK, Mueller S: Extrahepatic cholestasis increases liver stiffness (FibroScan) irrespective of fibrosis. Hepatology 2008, 48:1718-1723.

32. Harata M, Hashimoto S, Kawabe N, Nitta Y, Murao M, Nakano T, Arima Y, Shimazaki H, Ishikawa T, Okumura A, Ichino N, Osakabe K, Nishikawa T, Yoshioka K: Liver stiffness in extrahepatic cholestasis correlates positively with bilirubin and negatively with alanine aminotransferase. Hepatol Res 2011, 41:423-429.

33. Lebray P, Varnous S, Charlotte F, Varaut A, Poynard T, Ratziu V: Liver stiffness is an unreliable marker of liver fibrosis in patients with cardiac insufficiency. Hepatology 2089, 2008:48.

34. Poynard T, Munteanu M, Ngo Y, Castera L, Halfon P, Ratziu V, Imbert-Bismut F, Thabut D, Bourliere M, Cacoub P, Messous D, de Ledinghen V: ActiTest accuracy for the assessment of histological activity grades in patients with chronic hepatitis $C$, an overview using Obuchowski measure. Gastroenterol Clin Biol 2010, 34:388-396.

35. Yin M, Chen J, Glaser KJ, Talwalkar JA, Ehman RL: Abdominal magnetic resonance elastography. Top Magn Reson Imaging 2009, 20:79-87.

36. Wong F: Management of ascites in cirrhosis. J Gastroenterol Hepatol 2012, 27:11-20.

37. Ngo Y, Munteanu M, Messous D, Charlotte F, Imbert-Bismut F, Thabut D, Lebray P, Thibault V, Benhamou Y, Moussalli J, Ratziu V, Poynard T: A prospective analysis of the prognostic value of biomarkers (FibroTest) in patients with chronic hepatitis C. Clin Chem 2006, 52:1887-1896.

38. Poynard T, Ngo Y, Perazzo H, Munteanu M, Lebray P, Moussalli J, Thabut D, Benhamou $Y$, Ratziu V: Prognostic value of liver fibrosis biomarkers: a meta-analysis. Gastroenterol Hepatol (N Y) 2011, 7:445-454.

39. Palmeri ML, Wang MH, Dahl JJ, Frinkley KD, Nightingale KR: Quantifying hepatic shear modulus in vivo using acoustic radiation force. Ultrasound Med Biol 2008, 34:546-558.

40. Tozaki M, Isobe S, Fukuma E: Preliminary study of ultrasonographic tissue quantification of the breast using the acoustic radiation force impulse (ARFI) technology. Eur J Radiol 2011, 80:e182-e187.

41. Theise ND: Liver biopsy assessment in chronic viral hepatitis: a personal, practical approach. Mod Pathol 2007, 20(Suppl 1):S3-14.

42. Poynard T, Ratziu V, McHutchison J, Manns M, Goodman Z, Zeuzem S, Younossi Z, Albrecht J: Effect of treatment with peginterferon or interferon alfa- $2 \mathrm{~b}$ and ribavirin on steatosis in patients infected with hepatitis C. Hepatology 2003, 38:75-85.

43. Youden WJ: Index for rating diagnostic tests. Cancer 1950, 3:32-35.

44. Kim BH, Lee JM, Lee YJ, Lee KB, Suh KS, Han JK, Choi BI: MR elastography for noninvasive assessment of hepatic fibrosis: experience from a tertiary center in Asia. J Magn Reson Imaging 2011, 34:1110-1116.

45. Mueller S, Sandrin L: Liver stiffness: a novel parameter for the diagnosis of liver disease. Hepatic Medicine: Evidence and Research 2010, 2:49-65.

46. Motosugi U, Ichikawa T, Niitsuma Y, Araki T: Acoustic radiation force impulse elastography of the liver: can fat deposition in the liver affect the measurement of liver stiffness? Jpn J Radiol 2011, 29:639-643.

47. Yin M, Woollard J, Wang X, Torres VE, Harris PC, Ward CJ, Glaser KJ, Manduca A, Ehman RL: Quantitative assessment of hepatic fibrosis in an animal model with magnetic resonance elastography. Magn Reson Med 2007, 58:346-353.

48. Roulot D, Czernichow S, Le Clésiau H, Costes JL, Vergnaud AC, Beaugrand M: Liver stiffness values in apparently healthy subjects: influence of gender and metabolic syndrome. J Hepatol 2008, 48:606-613. 
49. Baba M, Furuya K, Bandou H, Kasai K, Sadaoka K: Discrimination of individuals in a general population at high-risk for alcoholic and nonalcoholic fatty liver disease based on liver stiffness: a cross section study. BMC Gastroenterol 2011, 11:70.

50. Talwalkar JA, Yin M, Venkatesh S, Rossman PJ, Grimm RC, Manduca A, Romano A, Kamath PS, Ehman RL: Feasibility of in vivo MR elastographic splenic stiffness measurements in the assessment of portal hypertension. Am J Roentgenol 2009, 193:122-127.

51. Wai CT, Greenson JK, Fontana RJ, Kalbfleisch JD, Marrero JA, Conjeevaram HS, Lok AS: A simple non-invasive index can predict both significant fibrosis and cirrhosis in patients with chronic hepatitis C. Hepatology 2003, 38:518-526.

52. Skripenova S, Trainer TD, Krawitt EL, Blaszyk H: Variability of grade and stage in simultaneous paired liver biopsies in patients with hepatitis C. J Clin Pathol 2007, 60:321-324.

53. Yang HI, Yuen MF, Chan HL, Han KH, Chen PJ, Kim DY, Ahn SH, Chen CJ, Wong W, Seto WK, REACH-B Working Group: Risk estimation for hepatocellular carcinoma in chronic hepatitis $B(\mathrm{REACH}-\mathrm{B})$ : development and validation of a predictive score. Lancet Oncol 2011, 12:568-574.

54. Kim SU, Kim Do Y, Park JY, Lee JH, Ahn SH, Kim JK, Paik YH, Lee KS, Chon $\mathrm{CY}$, Choi EH, Song KJ, Park YN, Han KH: How can we enhance the performance of liver stiffness measurement using FibroScan in diagnosing liver cirrhosis in patients with chronic hepatitis B? J Clin Gastroenterol 2010, 44:66-71.

55. Poynard T, Halfon P, Castera L, Munteanu M, Imbert-Bismut F, Ratziu V: Standardization of ROC curve areas for diagnostic evaluation of liver fibrosis markers based on prevalences of fibrosis stages. Clin Chem 2007, $53: 1615-1622$

doi:10.1186/1471-230X-12-105

Cite this article as: Chen et al:: Effects of patient factors on noninvasive liver stiffness measurement using acoustic radiation force impulse elastography in patients with chronic hepatitis C. BMC Gastroenterology 2012 12:105

\section{Submit your next manuscript to BioMed Central and take full advantage of:}

- Convenient online submission

- Thorough peer review

- No space constraints or color figure charges

- Immediate publication on acceptance

- Inclusion in PubMed, CAS, Scopus and Google Scholar

- Research which is freely available for redistribution 